\title{
Effects of micronutrient supplements on u.v.-induced skin damage
}

\author{
M. J. Jackson*, F. McArdle, A. Storey, S. A. Jones, A. McArdle and L. E. Rhodes $\dagger$ \\ Department of Medicine, University of Liverpool, Liverpool L69 3GA, UK
}

\begin{abstract}
Development of an orally-administered systemic agent that could reduce the effects of u.v. exposure on skin could potentially have a major effect on the incidence of skin cancers and photoageing. A number of micronutrients have been suggested to have metabolic properties that could induce this protection, and our data indicate that $n-3$ polyunsaturated fatty acids are particularly effective in this role. The mechanisms of action of $n-3$ polyunsaturated fatty acids appear to depend on their anti-inflammatory properties, acting to reduce the u.v.-induced release of cytokines and other mediators from a variety of skin cell types.
\end{abstract}

Polyunsaturated fatty acids: Anti-inflammatory: Antioxidant: Cytokines

Exposure of skin to u.v. radiation can result in both acute and long-term adverse effects on the skin. The acute effects include burning, photosensitivity and immunological alterations, and the longer-term effects can include photo-ageing and carcinogenesis (Taylor et al. 1990). The importance of these processes has been heightened by depletion of ozone in the atmosphere, leading to increased exposure of human subjects to u.v. radiation. (Frederick, 1993).

The mechanisms of $u . v$. radiation-induced inflammation are incompletely understood, but include increased production of prostaglandins and cytokines (Kupper et al. 1987). In addition, there is increasing evidence that exposure of cutaneous tissue to u.v. radiation leads to generation of free radical intermediates and reactive oxygen species (Black et al. 1978), potentially leading to peroxidation of membrane lipids and oxidative damage to DNA and proteins (Dixit et al. 1983; Punnonen et al. 1991).

Development of an effective systemic agent to reduce acute and chronic damage to skin due to u.v. radiation exposure could have important effects in reducing the increasing problems of premature photo-ageing of skin and skin cancers. There has been considerable interest in this area, particularly related to whether supplementation with nutritional antioxidants may influence the deleterious effects of u.v. radiation on skin (Nachbar \& Korting, 1995), but efficacy has not been firmly established.

Our group has undertaken a series of in vivo and in vitro studies designed to examine the potential of supplementation with either $n-3$ polyunsaturated fatty acids or common nutritional antioxidants to reduce the effects of u.v. radiation on skin cells. We reasoned that $n-3$ fatty acids could act in an anti-inflammatory manner to reduce the generation of prostaglandins and other inflammatory mediators by skin cells following u.v. exposure, while antioxidants were predicted to reduce effects of reactive oxygen species in damaging key components of the skin or inducing changes in gene expression regulating cell proliferation (Fig. 1).

\section{Polyunsaturated fatty acids}

Following some studies that indicated a potential role for fish oils in reduction of erythemal sensitivity to u.v. in hairless mice (Orengo et al. 1989), Orengo et al. (1992) reported preliminary data indicating that fish oil supplements reduced u.v. sensitivity in human subjects. In order to examine the potential efficacy of this approach, we examined the effect of supplementation with $10 \mathrm{~g}$ fish oil (containing $1.8 \mathrm{~g}$ eicosapentaenoic acid $(20: 5 n-3)$ and $1.2 \mathrm{~g}$ docosahexaenoic acid (22:6n-3), daily for 3 or 6 months in control subjects (Rhodes et al. 1994). Their erythemal responsiveness to u.v.B radiation was assessed at monthly intervals and at 10 weeks following cessation of supplements. In addition, paired biopsies of the epidermis were taken before and after 3 months of supplements. The dietary fish oil supplements increased the resistance of the skin to u.v.B-induced erythema, with more than doubling of a quantitative measure of sensitivity, the minimal erythemal dose. This response was associated with incorporation of $n-3$ polyunsaturated fatty acids into skin lipids, with pronounced increases in $20: 5 n-3$ and $22: 6 n-3$ contents.

Rhodes et al. (1994) speculated on the potential mechanisms by which this protection had occurred, suggesting that 


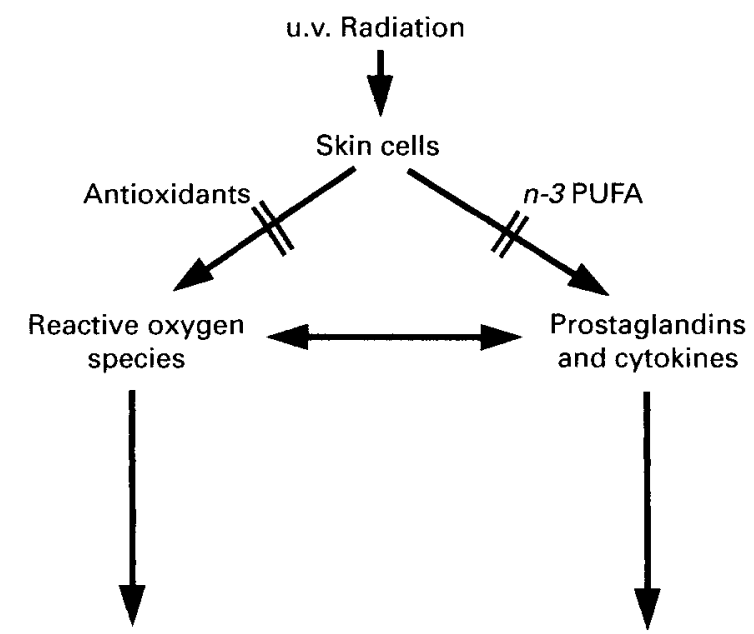

Acute damage, damage to DNA, changes in cell proliferation

Fig. 1. Schematic diagram to illustrate the potential complementary roles of $n-3$ polyunsaturated fatty acids (PUFA) and antioxidants in reducing $u . v$. radiation-induced skin damage.

a reduction in prostaglandin production, immune function or free radical production may be involved. In further work Rhodes et al. (1995) demonstrated a reduction in u.v. $B$-generated prostaglandin $E_{2}$ levels in the skin of fish oil-supplemented subjects, providing support for this potential mechanism of action. They also provided data in support of the use of these supplements in a light-sensitive disorder, polymorphic light eruption. An action of 20:5n-3 and/or 22:6n-3 to reduce u.v.-induced release of prostaglandin $\mathrm{E}_{2}$ or other inflammatory mediators is also supported by our recent data from keratinocyte and fibroblast culture studies (A Storey, L Rhodes and MJ Jackson, unpublished results).

\section{Nutritional antioxidants}

u.v. Irradiation of skin is associated with generation of a number of free radicals and reactive oxygen species, and some previous data indicate that antioxidants may play a protective role against u.v.-induced skin damage (De Rios et al. 1978; Hanada et al. 1991). Rhodes et al. (1994) suggested that one potential mechanism for the protective effect of fish oils was that the increased cellular content of oxidisable $n-3$ polyunsaturated fatty acids provides an oxidisable buffer protecting more vital structures against free radical damage. Such a mechanism had been proposed previously for the protective effect of fish oils against erythrocyte lipid peroxidation (van den Berg et al. 1991). No direct data for this possibility was provided, although epidermal lipid peroxidation was increased by the u.v. exposure.

As a preliminary to potential supplementation studies in vivo, we examined the effect of common nutritional antioxidants on u.v.-induced damage to skin fibroblasts (Jones et al. 1999). u.v. Radiation was found to induce a substantial oxidative stress in fibroblasts, resulting in increased release of superoxide anions and an increase in lipid peroxidation. Sub-lethal doses of u.v. radiation were also found to induce adaptive responses in the fibroblast antioxidant defences, with a transient rise in catalase and superoxide dismutase activities followed by a slower, larger, rise in cellular glutathione content. Supplementation of the fibroblasts with the antioxidants, Trolox (a water-soluble analogue of $\alpha$-tocopherol), ascorbic acid or $\beta$-carotene (all compounds obtained from Sigma, Poole, Dorset, UK), had differential effects on these responses (Jones et al. 1999). Trolox supplementation reduced the u.v. radiation-induced cellular oxidative stress and adaptive response in a predictable manner, whereas both ascorbate and $\beta$-carotene had less predictable effects. Both ascorbic acid and $\beta$-carotene increased the u.v. radiation-induced release of superoxide from the fibroblasts, but only at low doses were the adaptive responses in catalase and superoxide dismutase activities reduced.

These in vitro data appear to be generally supported by our recent unpublished studies in vivo (F McArdle, $\mathrm{L}$ Rhodes, P Friedmann and MJ Jackson, unpublished results), that show a reduction in u.v.-induced skin lipid peroxidation by vitamin $\mathrm{E}$ supplements. However, such changes were not associated with a reduction in erythemal sensitivity to u.v. in contrast to previously published data (De Rios et al. 1978).

\section{Conclusions}

Our data firmly support a role for supplementation with $n-3$ polyunsaturated fatty acids as a systemic approach to reduction of u.v. sensitivity in man. The mechanisms involved appear to primarily involve a reduction in u.v.-induced release of inflammatory mediators from skin cells. In contrast, little firm evidence for a beneficial role of supplementation with antioxidant nutrients has been obtained.

\section{Acknowledgements}

The authors would like to thank the World Cancer Research Fund, Ministry of Agriculture, Fisheries and Foods and the Medical Research Council for financial support, and their many co-workers and collaborators in this area.

\section{References}

Black AK, Greaves MW, Hensby CN \& Plummer NA (1978) Increased prostaglandins $E_{2}$ and $F_{2 \alpha}$ in human skin at 6 and 24 hour after ultraviolet $B$ irradiation. British Journal of Clinical Pharmacology 5, 431-436.

De Rios G, Chan JT, Black HS, Rudolph AH \& Knox JM (1978) Systemic protection by antioxidants against UVL-induced erythema. Journal of Investigative Dermatology 70, 123-125.

Dixit R, Mukhtar H \& Bickers DR (1983) Studies on the role of reactive oxygen species in mediating lipid peroxide formation in epidermal microsomes. Journal of Investigative Dermatology 81, 369-375.

Frederick JE (1993) Ultraviolet sunlight reaching the earth's surface: a review of recent research. Photochemistry and Photobiology 57, 175-178.

Hanada K, Gange RW \& Connor MJ (1991) Effect of gluathione depletion on sunburn cell formation in the hairless mouse. Journal of Investigative Dermatology 96, 838-840.

Jones SA, McArdle F, Jack CIA \& Jackson MJ (1999) Effect of antioxidant supplementation on the adaptive responses of human 
skin fibroblasts to UV-induced oxidative stress. Redox Report 4 , 291-299.

Kupper TS, Chua AO, Flood P, McGuire J \& Gubler U (1987) Interleukin 1 gene expression in cultured human keratinocytes is augmented by ultraviolet irradiation. Joumal of Clinical Investigation 80, 430-436.

Nachbar F \& Korting HC (1995) The role of vitamin E in normal and damaged skin. Journal of Molecular Medicine 73, 7-17.

Orengo IF, Black HS, Kettler AH \& Wolf JE (1989) Influence of dietary menhaden oil upon carcinogenesis and various cutaneous responses to ultraviolet radiation. Photochemistry and Photobiology 49, 71-77.

Orengo IF, Black HS \& Wolf JE (1992) Influence of fish oil supplementation on the minimal erythema dose in humans. Archives of Dermatological Research 284, 219-221.

Punnonen K, Autio P, Kiistala U \& Ahotupa M (1991) In-vivo effects of solar-simulated ultraviolet irradiation on antioxidant enzymes and lipid peroxidation in human epidermis. British Journal of Dermatology 125, 18-20.
Rhodes LE, Durham BH, Fraser WD \& Friedmann PS (1995) Dietary fish-oil reduces basal and ultraviolet B-generated PGE2 levels in skin and increases the threshold to provocation of polymorphic light eruption. Journal of Investigative Dermatology 105, 532-535.

Rhodes LE, O'Farrell S, Jackson MJ \& Friedmann PS (1994) Dietary fish-oil supplementation in humans reduces UVBerythemal sensitivity but increases epidermal lipid peroxidation. Joumal of Investigative Dermatology 103, 151-154.

Taylor DR, Stern RS, Leyden JJ \& Gilchrest BA (1990) Photoaging/photodamage and photoprotection. Journal of the American Academy of Dermatology 22, 1-15.

Van den Berg JJM, de Fouw NJ, Kuypers FA, Roelofsen B, Houtsmuller UMT \& Op den Kamp JAF (1991) Increased $n-3$ polyunsaturated fatty acid content of red blood cells from fish-oil fed rabbits increases in vitro lipid peroxidation, but decreases haemolysis. Free Radical Biology and Medicine 11, 393-399. 